\title{
The Outburst of the Blazar AO 0235+164 in 2006 December: Shock-in-Jet Interpretation
}

\author{
V.A. Hagen-Thorn ${ }^{1,4}$, V.M. Larionov ${ }^{1,2,4}$, S.G. Jorstad ${ }^{1,3}$, A.A. Arkharov ${ }^{1,2}$, E.I. \\ Hagen-Thorn ${ }^{1,2}$, N.V. Efimova ${ }^{1,2}$, L.V. Larionova ${ }^{1}$, A.P. Marscher ${ }^{3}$
}

\begin{abstract}
We present the results of polarimetric ( $R$ band) and multicolor photometric $(B V R I J H K)$ observations of the blazar AO $0235+16$ during an outburst in 2006 December. The data reveal a short timescale of variability (several hours), which increases from optical to near-IR wavelengths; even shorter variations are detected in polarization. The flux density correlates with the degree of polarization, and at maximum degree of polarization the electric vector tends to align with the parsec-scale jet direction. We find that a variable component with a steady power-law spectral energy distribution and very high optical polarization (30-50\%) is responsible for the variability. We interpret these properties of the blazar within a model of a transverse shock propagating down the jet. In this case a small change in the viewing angle of the jet, by $\lesssim 1^{\circ}$, and a decrease in the shocked plasma compression by a factor of $\sim 1.5$ are sufficient to account for the variability.
\end{abstract}

Subject headings: galaxies: active - galaxies: BL Lacertae objects: individual (AO 0235+164) — galaxies: jet — polarization

\section{Introduction}

Blazars are active galactic nuclei (AGNs) that possess extreme properties such as violent variability, high polarization, relativistic jets, and bright $\gamma$-ray emission. It is commonly

\footnotetext{
${ }^{1}$ Astronomical Institute of St. Petersburg State University, Universitetskiy Pr. 28, Petrodvorets, 198504, St. Petersburg, Russia; HTH-home@yandex.ru

${ }^{2}$ Main (Pulkovo) Astronomical Observatory of RAS, Pulkovskoe Sh. 60, 196140, St. Petersburg, Russia; vlar@astro.spbu.ru

${ }^{3}$ Institute for Astrophysical Research, Boston University, 725 Commonwealth Ave., Boston, MA 022151401; jorstad@bu.edu

${ }^{4}$ Isaac Newton Institute of Chile, St.-Petersburg Branch
} 
thought that the radio through optical emission in blazars is synchrotron radiation that originates in the jets. Polarimetric observations provide crucial data for investigating the magnetic field structure that forms the framework of such radiation. However, the polarization behavior is usually too complex for straightforward interpretation. For example, long-term observations of BL Lac reveal a change of degree of polarization from $0.5 \%$ to $>20 \%$ with all possible electric vector position angles (EVPAs), although the distribution of EVPAs indicates the existence of a preferential direction aligned with the parsec-scale jet (Hagen-Thorn et al. 1994). Difficulties are even more severe for physical models aimed to explain both the brightness and polarization behavior. A connection between polarization and brightness is rarely seen at optical wavelengths (Smith 1996). However, there are strong indications that the optical polarized emission is linked to the mm-wave VLBI core of blazars jets (Lister \& Smith 2000; Gabuzda et al. 2006; D'Arcangelo et al. 2007; Jorstad et al. 2007).

At radio wavelengths some progress in interpetation of both polarization and brightness variability has been achieved by using a model in which a shock propagates down the jet (e.g., Hughes, Aller, \& Aller 1989). At optical wavelengths, where polarization and brightness can vary on timescale of a few hours (e.g., Hagen-Thorn 1972; Miller \& Noble 1996; Carini 2006), a proper test of models requires very intensive monitoring of both polarization and brightness. Such simultaneous photometric and polarimetric measurements are difficult to obtain, but they provide a vital tool to distinguish among different models responsible for the variability.

We have observed the BL Lac object AO $0235+164 \quad(\mathrm{z}=0.94)$ during the relatively short outburst in 2006 December as part of the polarimetric and photometric monitoring program of blazars that has been carried out at Saint Petersburg State University for many years. AO $0235+164$ shows extreme blazar properties, such as intraday variability (e.g., Raiteri et al. 2005) and very high apparent speeds in the radio jet, $\beta_{\text {app }}=$ $(26 \pm 7) c$ (Piner et al. 2006), that have drawn the attention of many investigators to this object (Raiteri et al. 2001, 2005, 2006; Junkkarinen et al. 2004; Hagen-Thorn et al. 2007). In addition, AO $0235+164$ is one of the most highly polarized BL Lac objects known. During the outburst in 1983 January the optical polarization of the object varied from $12 \%$ to $24 \%$ over a 24 hour period (Smith 1996). Our polarimetric monitoring program has caught the source in a similar state but with an even higher degree of polarization, $\gtrsim 30 \%$. The polarimetric observations are enhanced by multicolor photometric measurements. Here we present the observations and show that the correlations that we have uncovered provide strong evidence in support of the shock model. 


\section{Observational data}

Observational data at optical wavelengths ( $B V R I$ bands) were obtained at the 70$\mathrm{cm}$ reflector of the Crimean Astrophysical Observatory with the photometer-polarimeter of the Astronomical Institute at St. Petersburg State University based on an ST-7 CCD. Methods of observation and data reduction of the photometric mode are described in detail in Hagen-Thorn et al. (2006). We used the comparison stars recommended by the Whole Earth Blazar Telescope (WEBT) program 1. The photometric errors do not exceed $0.03^{m}$ in $B$ band and $0.02^{m}$ in other bands when the object is brighter than $17^{m}$.

Observations at near-IR wavelengths ( $J H K$ bands) were carried out at the 1.1-m telescope of the Main (Pulkovo) Astronomical Observatory of the Russian Academy of Sciences located at Campo Imperatore (Italy) and equipped with the SWIRCAM camera. Methods of reduction are the same as for the optical observations. Errors in $J H K$ do not exceed $0.02^{m}$ when the object is brighter than $16^{m}$ in $\mathrm{J}$ band. In all cases the calibration of Mead et al. (1990) was used to transform magnitudes to flux densities (subsequently referred to as "fluxes").

Polarimetric observations were performed in $R$ band using two Savart plates rotated by $45^{\circ}$ relative to each another. By swapping the plates, the observer can obtain either the relative Stokes $q$ or $u$ parameter from the two split images of each source in the field. Instrumental polarization was found via stars located near the object under the assumption that their radiation is unpolarized. The Galactic latitude of AO $0235+164$ is $-39^{\circ}$ so that interstellar polarization (ISP) in this direction is less than $0.7 \%$. To correct for the ISP, the mean relative Stokes parameters of nearby stars were subtracted from the relative Stokes parameters of the object. This accounts for the instrumental polarization as well. The errors in degree of polarization are less than $2 \%$ for $R<16.5^{m}$, while the EVPA is determined with a precision of several degrees.

The results of photometric observations in the optical and IR bands are given in Tables 1 and 2, respectively. The light curves are shown in Figure 1 for the convenience of visual inspection. One can see that during the outburst the brightness increases by more than $3^{m}$. Table 3 lists the results of the polarimetric observations. In Figure 2 the upper panels illustrate the photometric $R$ and polarimetric observations for three consecutive Julian dates during the most dramatic period of the outburst. Intraday variations (IDV) are evident within a given Julian date. The bottom panels present absolute Stokes parameters during the same period. The existence of systematic variations during each night is a prominent

\footnotetext{
${ }^{1}$ http://www.to.astro/blazars/webt/
} 
feature of all the curves.

\section{Global Properties of Variability}

\subsection{Timescale of Variability}

We have determined the timescale of variability of the flux in each band using the definition proposed by Burbidge. Jones, \& O'Dell (1974): $\tau=d t / \ln \left(\mathrm{F}_{1} / \mathrm{F}_{2}\right)$, where $d t$ is the time interval between flux measurements $F_{1}$ and $F_{2}$, with $F_{1}>F_{2}$. We have calculated all possible timescales $\tau_{\mathrm{ij}}$ for any pair of observations for which $\left|F_{i}-F_{j}\right|>\sigma_{F_{i}}+\sigma_{F_{j}}$ at frequency $\nu$. The minimum timescale at frequency $\nu$ is determined as $\tau_{\nu}=\min \left\{\tau_{\mathrm{ij}, \nu}\right\}$, where $i=1, \ldots N-1, j=\mathrm{i}+1, \ldots N$, and $N$ is the number of observations. Table 4 lists the results of these calculations as well as the timescale of the polarized flux variability. The uncertainties of the values of $\tau_{\nu}$ are determined by errors of the flux measurements. However, the minimum timescale of variability that we derive is mainly constrained by the sampling, for example, the $B, V$, and $I$ light curves have only two measurements during JD 2454077 (Table 1), the date at which we find the minimum timescales in $R$ band and at IR wavelengths. In Table 4 we enclose the timescales derived from these light curves in brackets to indicate that they can not be compared with the timescales derived from the better sampled $R, J, H$, and $K$ light curves.

The values of $\tau_{\nu}$ indicate that during the event the timescale of variability was only a few hours and the shortest timescale, $\sim 0.3 \mathrm{hr}$, occured in the polarized flux. Comparison of the timescales of the total and polarized flux variability in $R$ band implies that the polarized emission originates in a subsection $(\sim 1 / 5)$ of the optical emission region. Moreover, the light curves with similar sampling during the event $(R, J, H$, and $K$ data) reveal an increase of timescale with wavelength.

\subsection{Multicolor Behavior}

We have calculated the discrete cross-correlation function, $D C F$, between the $R$ band light curve and those at other wavelengths using the approach suggested by Edelson \& Krolik (1988) for unequally spaced data. The functions were computed for lags between light curves from -6 to $+6 \mathrm{hr}$ with a step of $0.5 \mathrm{hr}$ and with the constraint that at least 5 pairs of measurements are needed to define the coefficient of correlation at a given delay. Figure 3 presents the results of the calculations. The absence of points at some lags indicates that the data are not capable of determining the $D C F$ for such delays owing to the constraint. 
Figure 3 shows that the $D C F$ s have maxima at different delays between the light curves. The maxima are distinct only for the optical light curves, and indicate that variations in $B$ band lead variations in $R$ band by $\sim 0.5-1 \mathrm{hr}$, while variations in $V$ and $I$ bands are simultaneous within $0.5 \mathrm{hr}$ with the variability in $R$ band. Although the peaks of the $D C F \mathrm{~s}$ for the near-IR light curves are broad and their positions are uncertain within 4-5 hrs, the delay of variations in the near-IR region compared to those in $R$ band is statistically significant. The peaks of the $D C F$ and their delays for each light curve with respect to the $R$ band light curve are listed in Table 4 . Such delays between the light curves are expected if the sizes of the emission regions at different wavelengths scale with the timescales of the variability found above.

\subsection{Polarization Properties}

Table 3 shows that very high degrees of polarization (up to 30\%) were observed during the December 2006 outburst. Figure 4 demonstrates that the degree of polarization increased when the object brightened. The latter is confirmed by the high value of the $D C F$ peak between the flux in $R$ band and the degree of polarization (Table 4). The DCF indicates also that the $R$ band and fractional polarization variations were simultaneous within $0.5 \mathrm{hr}$ (Fig. 3). Figure 4 shows a wide range, $\sim 90^{\circ}$, of variability of the polarization position angle. However, $\Theta_{\circ}$ aligns closely with the parsec jet direction, $\sim-15^{\circ}$ (Jorstad et al. 2001; Piner et al. 2006), when the degree of polarization exceeds $20 \%$.

Visual inspection of Figure 2 reveals a correlation between Stokes parameters - between $Q$ and $I$ and between $U$ and $I$ - and different behavior of the Stokes parameters from one epoch to another. The correlation coefficients calculated separately for each Julian date, given in Table 5, confirm the tight correlation between the Stokes parameters.

\section{Properties of the Source Responsible for Variability}

\subsection{Method of Analysis}

Our current understanding of active galactic nuclei pictures an AGN as a complex system in which different components - the host galaxy, accretion disk, corona above the disk, broad line regions, and jet - contribute to the total flux. In some cases multicolor photometric and polarimetric observations of variability may make it possible to derive the characteristics of the variable source (spectral energy distribution, SED, and polarization parameters) without knowledge of the absolute contribution of the other components to the total radiation. 
Let us suppose that the variability within some time interval is due to a single variable component. If the variability is caused only by its flux variation but the relative Stokes parameters $q$ and $u$ (for polarimetry) or relative SED (for photometry) remain unchanged, then in the space of absolute Stokes parameters $\{I, Q, U\}$ or in the $n$-dimensional flux space $\left\{F_{1}, \ldots, F_{n}\right\}$ ( $n$ is the number of spectral bands used in multicolor observations) the observational points must lie on straight lines. The slopes of these lines are relative Stokes parameters of the variable component (for polarimetry) or flux ratios for different pairs of bands as determined by its SED (for photometry).

With small caveats, the opposite is also true. In the case of photometry, a linear relation between observed fluxes in two different wavelengths during some period of flux variability implies that the flux ratio does not change. Such a relation for several bands indicates that the relative SED of the variable component remains steady and can be derived from the slopes of the lines. In the case of polarimetry, a linear relation between observed Stokes parameters suggests that the variable component has constant degree of polarization and polarization position angle, the values of which can be obtained from the relative Stokes parameters $q$ and $u$ that correspond to the slope of the $Q$ vs. $I$ and $U$ vs. $I$ lines, respectively. Further details of the method of analysis are provided in Hagen-Thorn \& Marchenko (1999).

\subsection{Polarization Parameters of the Variable Component}

Correlation plots of the absolute Stokes parameters for three consecutive epochs during the outburst (JD 2454077, 2454078, and 2454079) are given in Figure 5 (a,b, and c, respectively). One can see that in all cases the points lie on straight lines that leads us to conclude that during a given epoch the relative Stokes parameters of the variable component remained unchanged. The slopes of the straight lines in Figure 5 are determined by the orthogonal regression method. They give the relative Stokes parameters $q_{v a r}$ and $u_{v a r}$ of the variable component that we use to calculate its degree and position angle of polarization, $p_{v a r}$ and $\Theta_{v a r}$ (Table 5). Table 5 indicates that the degree of polarization of the variable component is very high and decreases from $50 \%$ to $30 \%$ as the object fades. This is accompaned by a systematic variation of the polarization direction. At the highest degree of polarization the EVPA tends to align to within $15^{\circ}$ with the direction of the parsec-scale jet inferred from the VLBI maps of AO 0235+164 (Jorstad et al. 2001; Piner et al. 2006). 


\subsection{Spectral Energy Distribution of the Variable Component}

We compare the fluxes in different bands relative to the $R$ and $J$ fluxes for the optical and near-IR data, respectively (see Fig. 6), since these two bands have the largest number of observations. According to Figure 2, the data reveal intraday variability that can affect the flux-flux comparison because the measurements are not completely simultaneous. For example, during Julian dates with intensive monitoring, delays of $\sim 10-15$ minutes between observations in different bands occur. For such epochs the fluxes in $R$ band were interpolated to the corresponding times of the $B, V$, and $I$ bands measurements (the same for $J$ band with respect to $H$ and $K$ bands). For epochs with a few measurements, the average at each band was used.

The slopes of the lines were derived using the orthogonal regression method. They are corrected for extinction based on the information provided by Raiteri et al. (2005). The logarithms of the corrected values are listed in Table 4. Using these values, we have constructed SEDs in the optical and near-IR regions as well as the combined SED (via the flux-flux diagram for $R$ and $J$ ) for the whole range from $B$ to $K$ bands (Fig. 7). Figure 7 shows that the spectra can be represented by a power law, $F_{\nu} \sim \nu^{-\alpha}$, where $\alpha$ is the spectral index. In the near-IR region $\alpha=1.10 \pm 0.03$, while in the optical region $\alpha=1.39 \pm 0.12$. Although there appears to be a slight steepening of the spectrum in the optical region, this falls within the $2 \sigma$ uncertainty, so that the combined SED can be fit by a single power law with $\alpha=1.04 \pm 0.06$. This indicates that the same component with the relative SED shown in Figure 7 is responsible for the source variability in the whole range from $B$ to $K$ bands.

\section{Discussion}

The results of the polarimetric and photometric observations shows that the dramatic variability seen in AO 0235+164 in 2006 December might be explained by a single variable component that possesses the following properties: (i) steady spectral shape; (ii) increase of size of the emitting region with wavelength; (iii) very high degree of polarization, up to $50 \%$; (iv) strong correlation between degree of polarization and flux; and (v) tendency of the magnetic field at the highest polarization state to be perpendicular to the inner radio jet direction. These properties are characteristics of the model that describes an outburst in a blazar as a transverse shock propagating down a relativistic jet with turbulent magnetic

field (Marscher \& Gear 1985; Hughes, Aller, \& Aller 1985; Marscher 1996). The timescale of variability relates to the thickness of the shock front, which is determined by the lifetime of relativistic electrons accelerated at the front. This thickness decreases with frequency owing to synchrotron radiation losses. The shock orders the turbulent magnetic field along 
the front, which is perpendicular to the jet direction.

We consider a model of a square-wave transverse shock moving through a turbulent plasma with a constant Lorentz factor, $\Gamma$. The size and shape of the emission region at a given frequency are steady. In the observer's frame the flux of the shocked region is boosted as follows:

$$
F=F_{\circ} \nu^{-\alpha} \delta^{(2+\alpha)} \delta^{(3+\alpha)},
$$

where $\delta^{\prime}$ is the Doppler factor of the shocked plasma in the rest frame of the shock front and $\delta$ is the Doppler factor of the shock front in the observer's frame. Determination of $\delta^{\prime}$ requires knowledge of the speed and equation of state of the plasma behind the shock front (Cawthorne 1991), which is difficult to specify due to a lack of the necessary information. Instead, we assume that the velocity of the shocked plasma in the frame of the shock front $\ll c$ and $\delta^{\prime} \approx 1$. We can then approximate that $\delta=[\Gamma(1-\beta \cos \Phi)]^{-1}$, where $\beta=\sqrt{1-\Gamma^{-2}}$ is the speed of the shock and $\Phi$ is the angle between the line of sight and the velocity of the centroid of the emission region.

The degree of polarization depends on the viewing angle of the shock, $\Psi$, and ratio of the density in the shocked region with respect to unshocked region, $\eta=n_{\text {shock }} / n_{\text {unshock }}$ (Hughes \& Miller 1991):

$$
p \approx \frac{\alpha+1}{\alpha+5 / 3} \frac{\left(1-\eta^{-2}\right) \sin ^{2} \Psi}{2-\left(1-\eta^{-2}\right) \sin ^{2} \Psi} .
$$

The viewing angle of the shock in the observer's frame is subjected to relativistic aberration and determined by the bulk Lorentz factor and viewing angle of the jet:

$$
\Psi=\tan ^{-1}\left[\sin \Phi /\left(\Gamma\left(\cos \Phi-\sqrt{1-\Gamma^{-2}}\right)\right)\right]
$$

We use the derived spectral index of the variable component $\alpha=1.04$ and assume $\Gamma=30$, consistent with the highest reliable apparent speed seen in the jet of AO $0235+16$ (Jorstad et al. 2001; Piner et al. 2006). We apply equation (1) to the photometric data in the $R$ band to estimate the Doppler factor as a function of time. The scaling factor $F_{\circ}$ is determined under the assumption that $F_{\circ}=F_{\max } \nu^{\alpha} / \delta_{\circ}^{(3+\alpha)}$, where $F_{\max }$ is the maximum observed flux in $R$ band and $\delta_{\circ}$ is obtained for $\Phi_{\circ}$, which is calculated from equations (2) and (3) for the maximum derived degree of polarization of the variable component (Table 51). In this case we adopt $\eta=2.5$ that is the minimum possible compression that produces polarization as high as $50 \%$. This yields $\delta_{\circ}=23.3$ at the maximum polarization. Figure 8 shows the values of the Doppler factor and viewing angle of the jet derived from the observed flux variability. The latter allows us to estimate the viewing angle of the shock (eq. 3) and 
value of the plasma compression as functions of time using the light curve of fractional polarization (eq. 2). The values of $\Psi$ and $\eta$ are shown in Figure 8 as well.

Figure 8 demonstrates that the observed flux and polarization behavior can be explained by a small change in the viewing angle $\lesssim 1^{\circ}$ and by variations of shocked plasma compression while the bulk Lorentz factor remains constant. A small viewing angle $\left(\sim 2.5^{\circ}\right)$ agrees with the highly core-dominated radio images of the blazar. A change in the viewing angle can be caused either by curvature of the jet by $\lesssim 1^{\circ}$ or by a curved trajectory of the shock-causing perturbation inside the jet for an opening angle $\gtrsim 1^{\circ}$. In the brightest flux state the viewing angle is the nearest to the line of sight, the shock is seen almost edge-on $\left(\Psi \sim 103^{\circ}\right)$, the plasma compression is the highest $(\eta \sim 1.7)$, and the parameters change on timescale of hours. In the end of the outburst $\eta \sim 1.1$ and the parameters are steady within a week. Changes in the rate of injection of relativistic electrons could be related to variations in $\eta$.

In this scenario a shock is responsible for the injection of the relativistic electrons into the emitting region, leading to a steady spectral shape and a short timescale of the variability in the optical and IR bands. Therefore, we assume that the total flux and linear polarization variability is caused by changes in the viewing angle and in injection of relativistic electrons on timescales of hours. Radiative losses determine the size of the emitting region behind the shock front, which is of the order of 2-3 light hours (Table 4). A steady spectral shape implies a quasi-steady state of the emission, which is re-established every 2-3 hrs, between the rate of injection and the radiative losses. In the observer's frame the synchrotron lifetime of electrons emitting at frequency $\nu$ is:

$$
t_{\mathrm{loss}} \approx 4.75 \times 10^{2}\left[\frac{1+z}{\delta \nu_{G H z} B_{G}^{3}}\right]^{1 / 2} \text { days }
$$

where $B_{G}$ is the magnetic field in Gauss. Since $t_{\text {loss }} \approx \tau_{\nu}$, for $\delta=23.3$ (the maximum derived Doppler factor) and $\tau_{\nu}=1.7 \mathrm{hr}$ in the $R$ band (Table 4), eq.(4) yields an estimate of the magnetic field, $B \approx 0.5 \mathrm{G}$, that agrees with other estimates of the typical magnetic field in a blazar core (e.g., Marscher \& Gear 1985). Equation (4) implies also that the timescale of variability in $K$ band should be longer than that in $R$ band by a factor of 1.8 , consistent with the values of $\tau_{\nu}$ derived from the observations. The highest degree of polarization, $p_{\text {var }}=50 \%$, is close to the maximum possible polarization $(75 \%)$ for a synchrotron source with $\alpha=1.04$ and a uniform magnetic field (Pacholczyk 1970). This indicates an extremely well ordered magnetic field behind the shock front along the plane transverse to the jet direction. Therefore, we consider that the polarimetric and multicolor photometric behavior of AO $0235+164$ during the outbust in 2006 December is consistent with the properties of the shock-in-jet model. 


\section{Conclusion}

In 2006 December the BL Lac object AO 0235+164 underwent a spectacular outburst with an increase in optical flux by a factor of 4 and with degree of polarization reaching $30 \%$. Analysis of the multicolor photometric data and polarization measurements in $R$ band indicate that:

(i) The flux increase has an achromatic character.

(ii) The spectral energy distribution of the variable component is a power law with $\alpha=1.04 \pm 0.06$.

(iii) Variations on timescales of hours are present at all wavelengths. The minimum timescale of variability increases with wavelength.

(iv) There is a delay between the variations at different wavelengths, with fluctuations occurring first in $B$ band and last at the near-IR wavelengths.

(v) The fractional polarization correlates with the flux.

(vi) The direction of polarization at the highest polarization states tends to align with the inner radio jet direction.

These properties of the variable component responsible for the outburst match the characteristics of a transverse shock propagating down the jet. We find that the observed

photometric and polarimetric variability can be reproduced in detail by the shock propagating down a twisted jet such that the viewing angle changes by $\lesssim 1^{\circ}$ and the plasma compression decreases by a factor of $\sim 1.5$ while the bulk Lorentz factor, $\Gamma=30$, remains constant.

This work is supported by the Russian Fund of Basic Research, grant 05-02-17562. S. Jorstad and A. Marscher acknowledge support from the National Science Foundation under grant no. AST-0406865.

\section{REFERENCES}

Burbidge, G. R., Jones, T. W., \& O’Dell, S. L. 1974, ApJ, 193, 43

Carini, M. 2006, in Blazar Variability Workshop II: Entering the GLAST Era, ed. H.R. Miller, K. Marshall, J.R. Webb, \& M.F. Aller, ASP Conference Series, 350, 55 
Cawthorne, T. V. 1991, in Beams and Jets in Astrophysics, ed. P. A. Hughes, Cambridge Astrophysics Series, (Cambridge Univ. Press), 187

D'Arcangelo, F. D. et al. 2007, ApJ, 659, L107

Edelson, R. A., \& Krolik, J. H. 1988, ApJ, 333, 646

Gabuzda, D. C., Rastorgueva, E. A., Smith, P. S., \& O’Sullivan, S. P. 2006, MNRAS, 369, 1596

Hagen-Thorn, V. A. 1972, Astron. Circ., 714, 5 (in Russian)

Hagen-Thorn, V. A. \& Marchenko, S. G. 1999, Baltic Astron., 8, 575

Hagen-Thorn, V. A., Marchenko, S. G., Yurchenko, A. V., Yakovleva, V. A. 1994, Astron. Reports, 38, 635

Hagen-Thorn, V. A., et al. 2006, Astron. Reports, 50, 458

Hagen-Thorn, V. A., et al. 2007, Astron. Reports, in press

Hughes, P. A., Aller, H. D., \& Aller, M. F. 1985, ApJ, 298, 301

Hughes, P. A., Aller, H. D., \& Aller, M. F. 1989, ApJ, 341, 68

Hughes, P. A., \& Miller, L. 1991, in Beams and Jets in Astrophysics, ed. P. A. Hughes, Cambridge Astrophysics Series, (Cambridge Univ. Press), 1

Jorstad, S. G., et al. 2001, ApJS, 134, 181

Jorstad, S. G., et al. 2007, AJ, 134, 799

Junkkarinen, V. T., et al. 2004, ApJ, 614, 658

Lister, M. L., \& Smith, P. S. 2000, ApJ, 541, 66

Marscher, A. P., \& Gear, W. K. 1985, ApJ, 298, 114

Marscher, A. P. 1996, in Blazar Continuum Variability, ed. H.R. Miller, J.R. Webb, \& J.C. Noble, ASP Conference Series, 110, 248

Mead, A. R. J., et al. 1990, A\&AS, 83, 183

Miller, H. R., \& Noble, J. C. 1996, in Blazar Continuum Variability, ed. H.R. Miller, J.R. Webb, \& J.C. Noble, ASP Conference Series, 110, 17 
Pacholczyk, A. G., 1970, Radio Astrophysics (W.H. Freeman and Company, San Francisco)

Piner, B. G., Bhattarai, D., Edwards, P. G., \& Jones, D. L. 2006, ApJ, 640, 196

Raiteri, C. M., et al. 2001, A\&A, 377, 396

Raiteri, C. M., et al. 2005, A\&A, 438, 39

Raiteri, C. M., et al. 2006, A\&A, 459, 731

Smith, P. S. 1996, in Blazar Continuum Variability, ed. H.R. Miller, J.R. Webb, \& J.C. Noble, ASP Conference Series, 110, 135 
Table 1. Optical Data

\begin{tabular}{|c|c|c|c|c|c|c|c|}
\hline $\begin{array}{l}\text { MJD } \\
(1)\end{array}$ & $\begin{array}{c}B \\
(2)\end{array}$ & $\begin{array}{l}\text { MJD } \\
(3)\end{array}$ & $\begin{array}{c}V \\
(4)\end{array}$ & $\begin{array}{l}\text { MJD } \\
(5)\end{array}$ & $\begin{array}{c}R \\
(6)\end{array}$ & $\begin{array}{c}\text { MJD } \\
(7)\end{array}$ & $\begin{array}{c}I \\
(8)\end{array}$ \\
\hline 27.3865 & $20.26 \pm 0.17$ & 27.3946 & $19.06 \pm 0.05$ & 27.3784 & $18.43 \pm 0.03$ & 27.3823 & $17.65 \pm 0.03$ \\
\hline 28.3267 & $19.71 \pm 0.17$ & 28.3345 & $19.01 \pm 0.07$ & 28.3489 & $18.44 \pm 0.04$ & 28.3473 & $17.63 \pm 0.03$ \\
\hline 29.3514 & $19.95 \pm 0.17$ & 29.3592 & $19.08 \pm 0.05$ & 29.3749 & $18.45 \pm 0.04$ & 29.3735 & $17.68 \pm 0.03$ \\
\hline
\end{tabular}

Note. - MJD is JD-2454000; the table appears in full form electronically.

Table 2. Near-IR Data

\begin{tabular}{|c|c|c|c|c|c|}
\hline $\begin{array}{c}\text { MJD } \\
(1)\end{array}$ & $\begin{array}{c}J \\
(2)\end{array}$ & $\begin{array}{c}\text { MJD } \\
(3)\end{array}$ & $\begin{array}{c}H \\
(4)\end{array}$ & $\begin{array}{c}\text { MJD } \\
(5)\end{array}$ & $\begin{array}{c}K \\
(6)\end{array}$ \\
\hline 979.3865 & $17.24 \pm 0.11$ & 979.5627 & $16.45 \pm 0.15$ & 979.5666 & $15.31 \pm 0.19$ \\
\hline 980.3267 & $17.25 \pm 0.07$ & 980.6206 & $16.32 \pm 0.09$ & 980.6258 & $15.47 \pm 0.16$ \\
\hline 985.3514 & $17.27 \pm 0.06$ & 985.5778 & $16.19 \pm 0.05$ & 985.5828 & $15.70 \pm 0.16$ \\
\hline
\end{tabular}

Note. - MJD is JD-2453000; the table appears in full form electronically.

Table 3. Polarization Data in $R$ band

\begin{tabular}{ccc}
\hline \hline $\begin{array}{c}\text { MJD } \\
(1)\end{array}$ & $\begin{array}{c}\text { p, } \\
(2)\end{array}$ & $\begin{array}{c}\Theta^{\circ} \\
(3)\end{array}$ \\
\hline 77.3307 & $32.7 \pm 1.0$ & $149.5 \pm 0.8$ \\
77.4282 & $27.7 \pm 1.4$ & $152.2 \pm 1.3$ \\
77.4433 & $25.9 \pm 1.6$ & $153.8 \pm 1.8$ \\
\hline
\end{tabular}

Note. - Columns are as follows: (1) - JD-2454000; (2) - degree of the polarization; (3) - position angle of the polarization; the table appears in full form electronically. 
Table 4. Multicolor Properties of the Variable Component

\begin{tabular}{|c|c|c|c|c|c|c|}
\hline $\begin{array}{l}\text { Band } \\
(1)\end{array}$ & $\begin{array}{c}\lg (\nu) \\
(2)\end{array}$ & $\begin{array}{l}N \\
(3)\end{array}$ & $\begin{array}{c}\tau_{\min }, \mathrm{hr} \\
\quad(4)\end{array}$ & $\begin{array}{c}D C F_{\text {peak }} \\
(5)\end{array}$ & $\begin{array}{c}\Delta t, \mathrm{hr} \\
(6)\end{array}$ & $\begin{array}{c}\lg \left(F_{\nu} / F_{R}\right) \\
(7)\end{array}$ \\
\hline$B$ & 14.833 & 24 & $\left(5.27_{-0.90}^{+1.36}\right)$ & $0.89 \pm 0.10$ & $+1.0_{-1}^{+0.5}$ & $-0.211 \pm 0.012$ \\
\hline V & 14.736 & 24 & $\left(6.15_{-0.34}^{+0.40}\right)$ & $0.95 \pm 0.11$ & $0.0_{-0.5}^{+1}$ & $-0.120 \pm 0.006$ \\
\hline$R$ & 14.670 & 66 & $1.73_{-0.29}^{+0.22}$ & 1.0 & 0.0 & 0.0 \\
\hline$I$ & 14.574 & 24 & $\left(4.81_{-0.25}^{+0.29}\right)$ & $0.85 \pm 0.09$ & $0.0_{-0.5}^{+1}$ & $0.142 \pm 0.007$ \\
\hline$J$ & 14.387 & 55 & $2.91_{-0.34}^{+0.46}$ & $0.98 \pm 0.02$ & $-3.5_{-2.5}^{+1.5}$ & $0.256 \pm 0.007$ \\
\hline$H$ & 14.262 & 55 & $2.19_{-0.37}^{+0.55}$ & $0.98 \pm 0.03$ & $-3.5_{-2.5}^{+1.5}$ & $0.400 \pm 0.006$ \\
\hline$K$ & 14.140 & 54 & $3.01_{-0.67}^{+1.21}$ & $0.97 \pm 0.03$ & $-3.5_{-2.5}^{+1.5}$ & $0.526 \pm 0.005$ \\
\hline$P$ & 14.670 & 54 & $0.31_{-0.04}^{+0.04}$ & $0.74 \pm 0.08$ & $0.0_{-1.5}^{+1}$ & $\ldots$ \\
\hline
\end{tabular}

Note. - Columns are as follows: (1) - filter of photometry, $P$ corresponds to the polarization data; (2) - logarithm of effective frequency of the filter; (3) - number of observations; (4) - minimum timescale of variability, unreliable values are enclosed in brackets; (5) - peak of discrete cross-correlation function of the light curve at frequency $\nu$ with respect to $R$ band light curve; in the case of polarization data the peak is given for the $D C F$ between the $R$-band and fractional polarization light curves; $(6)$ - delay of the peak; + means that the light curve at frequency $\nu$ leads the $R$ band light curve, while - is the opposite case; (7) - logarithm of the contribution of the variable component in the SED at frequency $\nu$ relative to the contribution in $R$ band.

Table 5. Polarization Properties of the Variable Component

\begin{tabular}{crrcc}
\hline \hline $\begin{array}{c}\text { MJD } \\
(1)\end{array}$ & $\begin{array}{r}r_{Q I} \\
(2)\end{array}$ & $\begin{array}{r}r_{U I} \\
(3)\end{array}$ & $\begin{array}{c}p_{\text {var }}, \% \\
(4)\end{array}$ & $\begin{array}{c}\Theta_{\text {var }},{ }^{\circ} \\
(5)\end{array}$ \\
\hline 77.447 & 0.93 & -0.90 & $50.0 \pm 9.4$ & $-30.4 \pm 5.4$ \\
78.373 & -0.48 & -0.98 & $36.7 \pm 3.4$ & $-49.8 \pm 2.7$ \\
79.320 & -0.58 & -0.92 & $33.1 \pm 5.2$ & $-53.9 \pm 4.5$ \\
\hline
\end{tabular}

Note. - Columns are as follows: (1) - Julian date minus 2454000; (2) - correlation coefficient between Stokes parameters $Q$ and $I ;(3)$ - correlation coefficient between Stokes parameters $U$ and $I ;(4)$ - degree of polarization; (5) - position angle of polarization. 


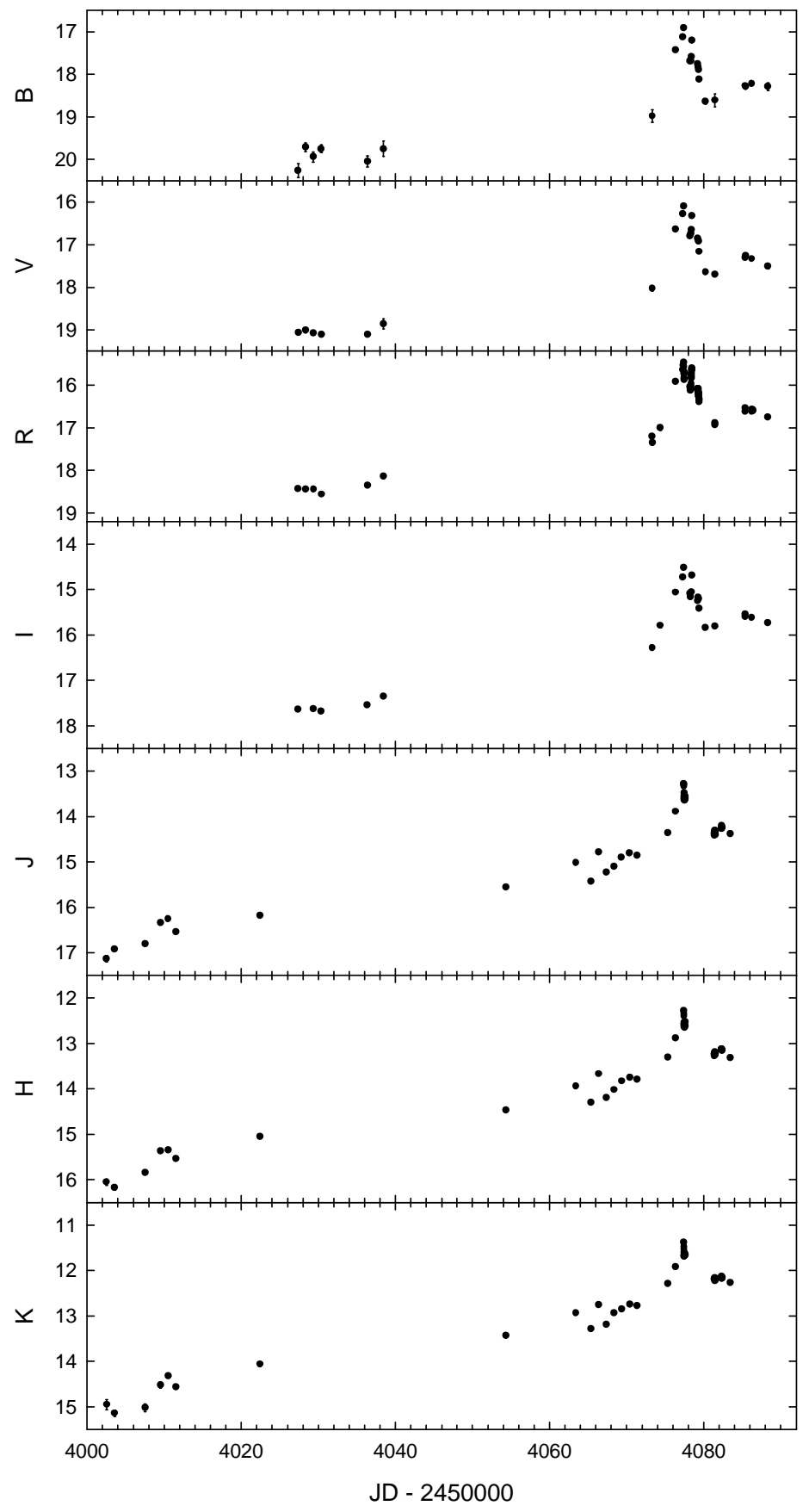

Fig. 1. - Light curves of AO $0235+16$ in the optical and IR bands. 


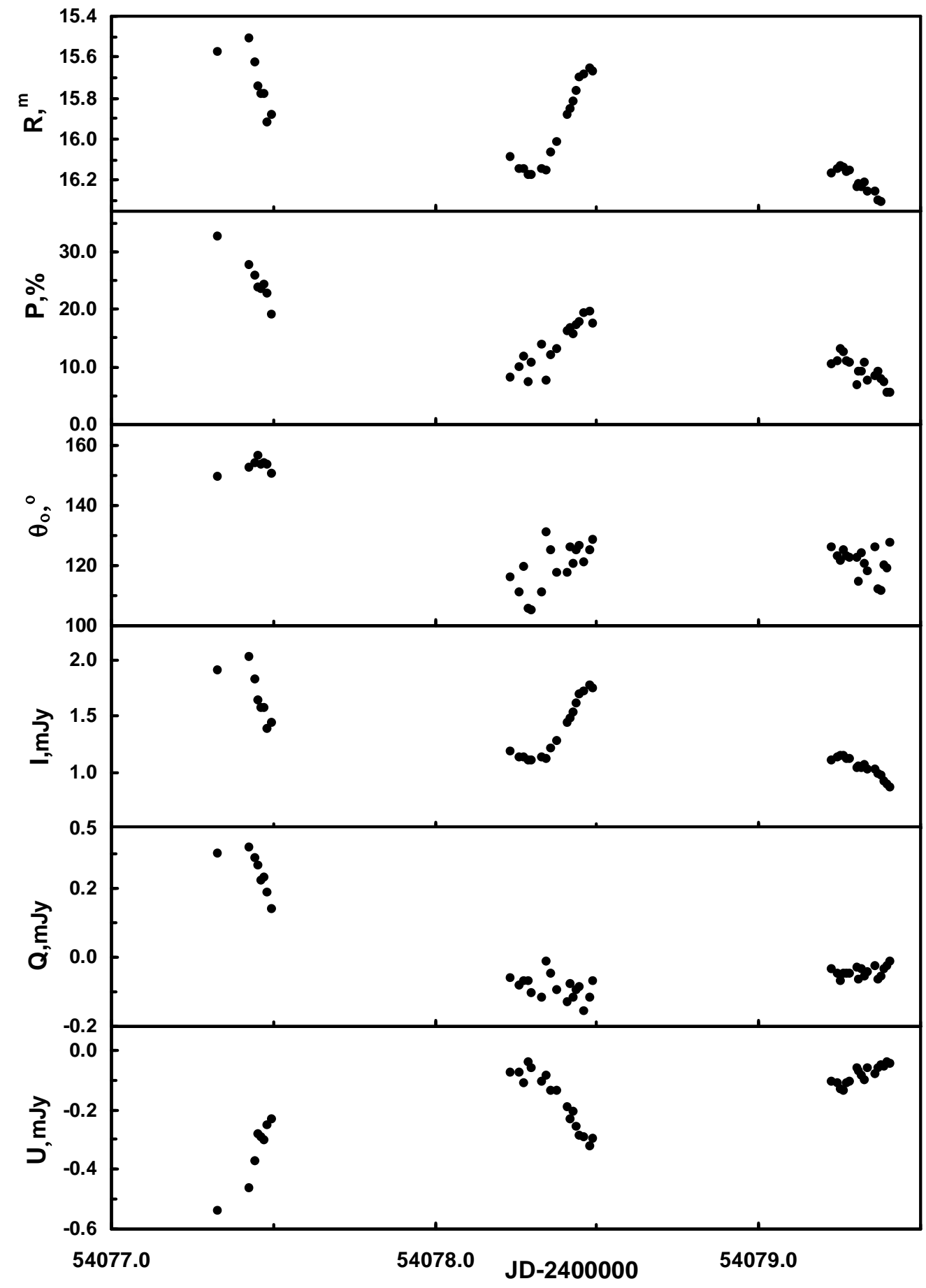

Fig. 2.- Simultaneous photometric and polarimetric data for AO $0235+16$ at three consecutive Julian dates during the outburst. 


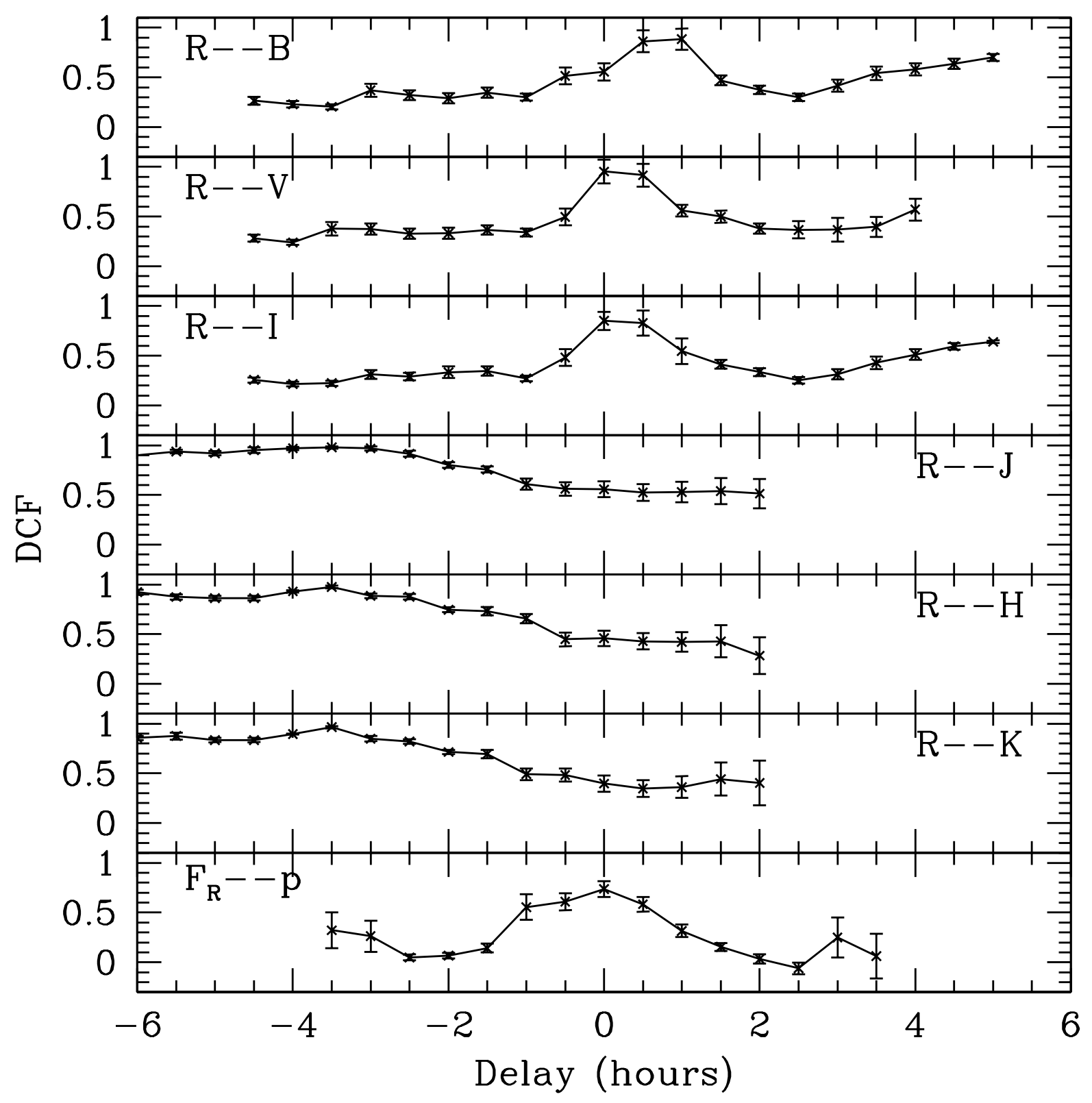

Fig. 3.- Discrete cross-correlation function between light curves in different bands with respect to the $R$-band light curve; a negative delay means that $R$ band variations lead. Bottom panel: DCF between degree of polarization and total flux density in $R$ band. 


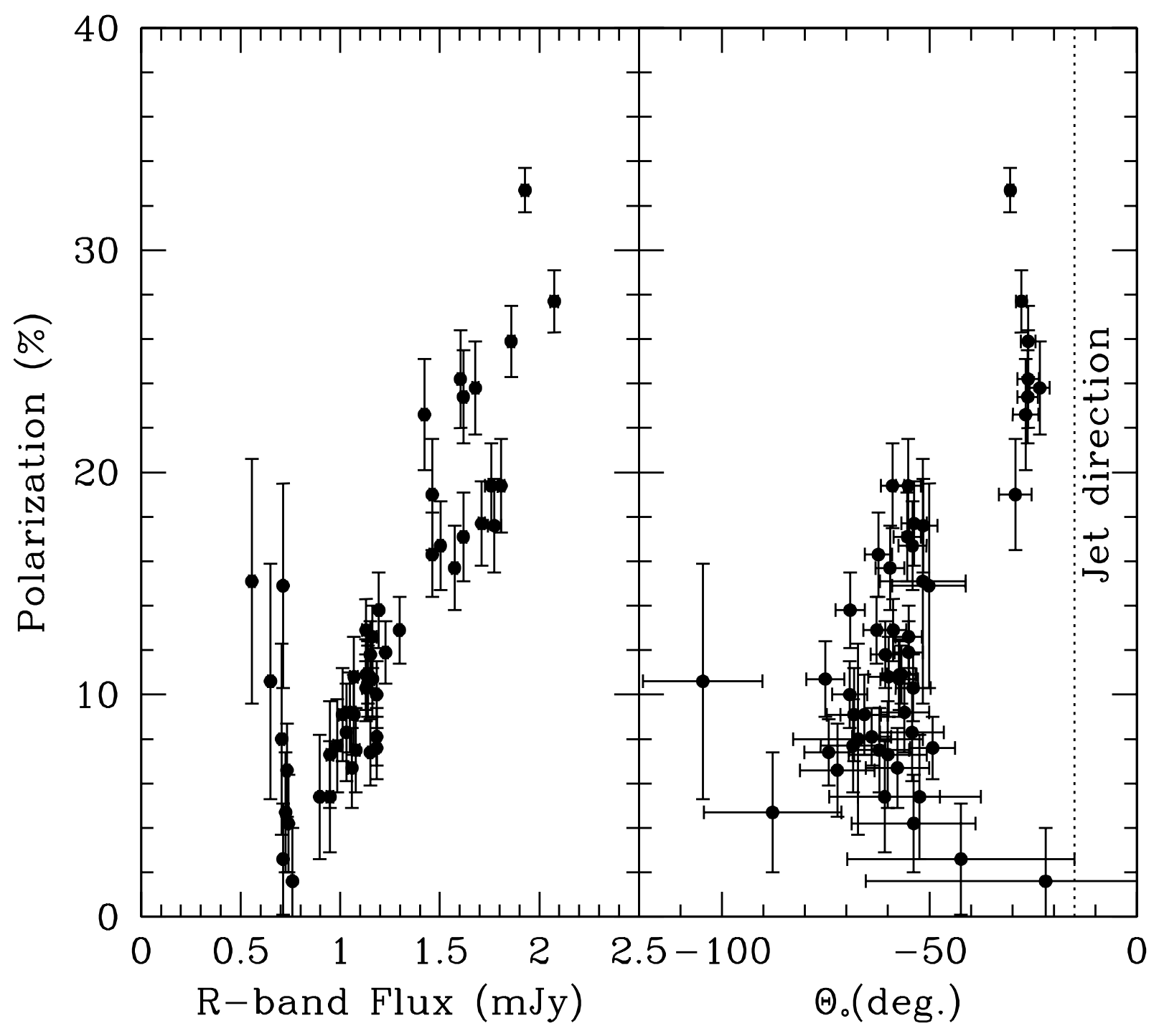

Fig. 4.- Left panel: Dependence between the degree of polarization and flux in $R$ band. Right panel: Dependence between the degree of polarization and polarization position angle; the dotted line indicates direction of the parsec scale jet. 

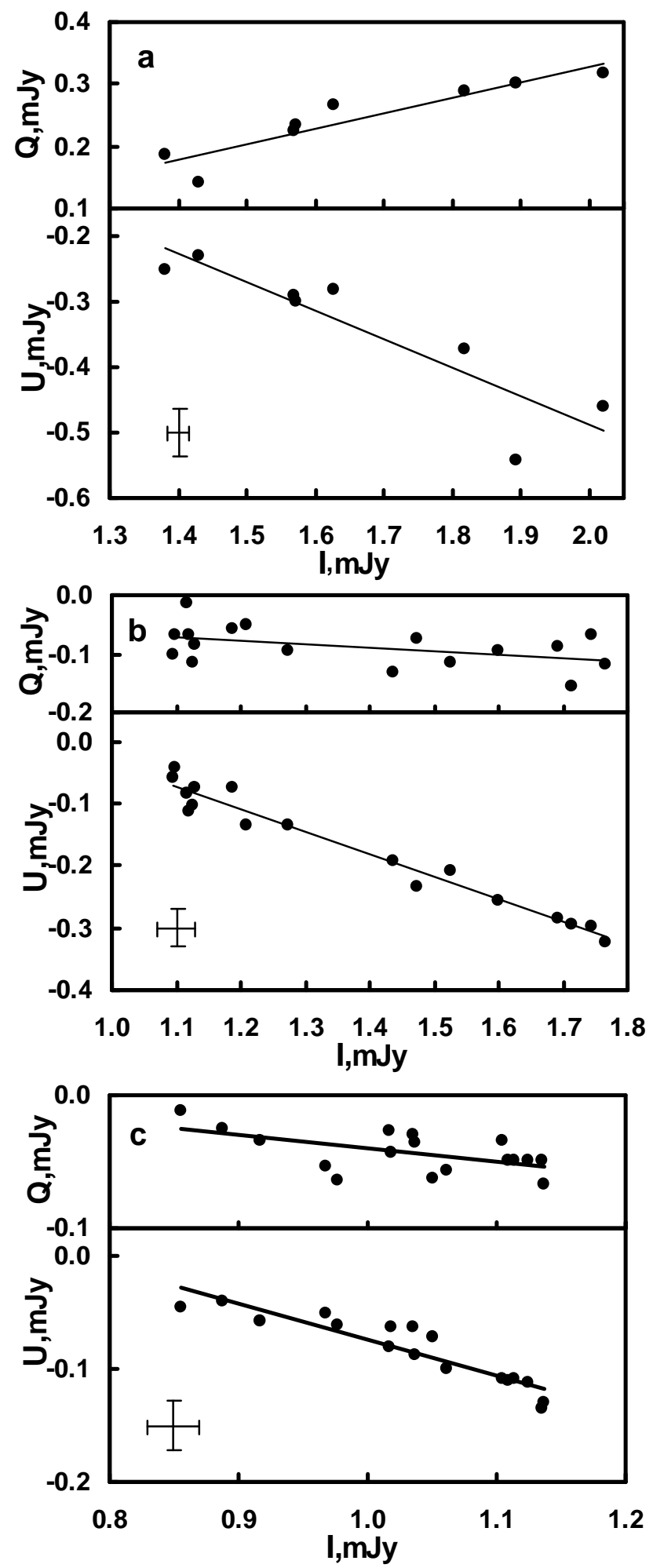

Fig. 5.- Comparison of absolute Stokes parameters for three consecutive Julian dates during the outburst (a - 2454077, b - 2454078, c - 2454079); crosses correspond to the uncertainties of the parameters. 

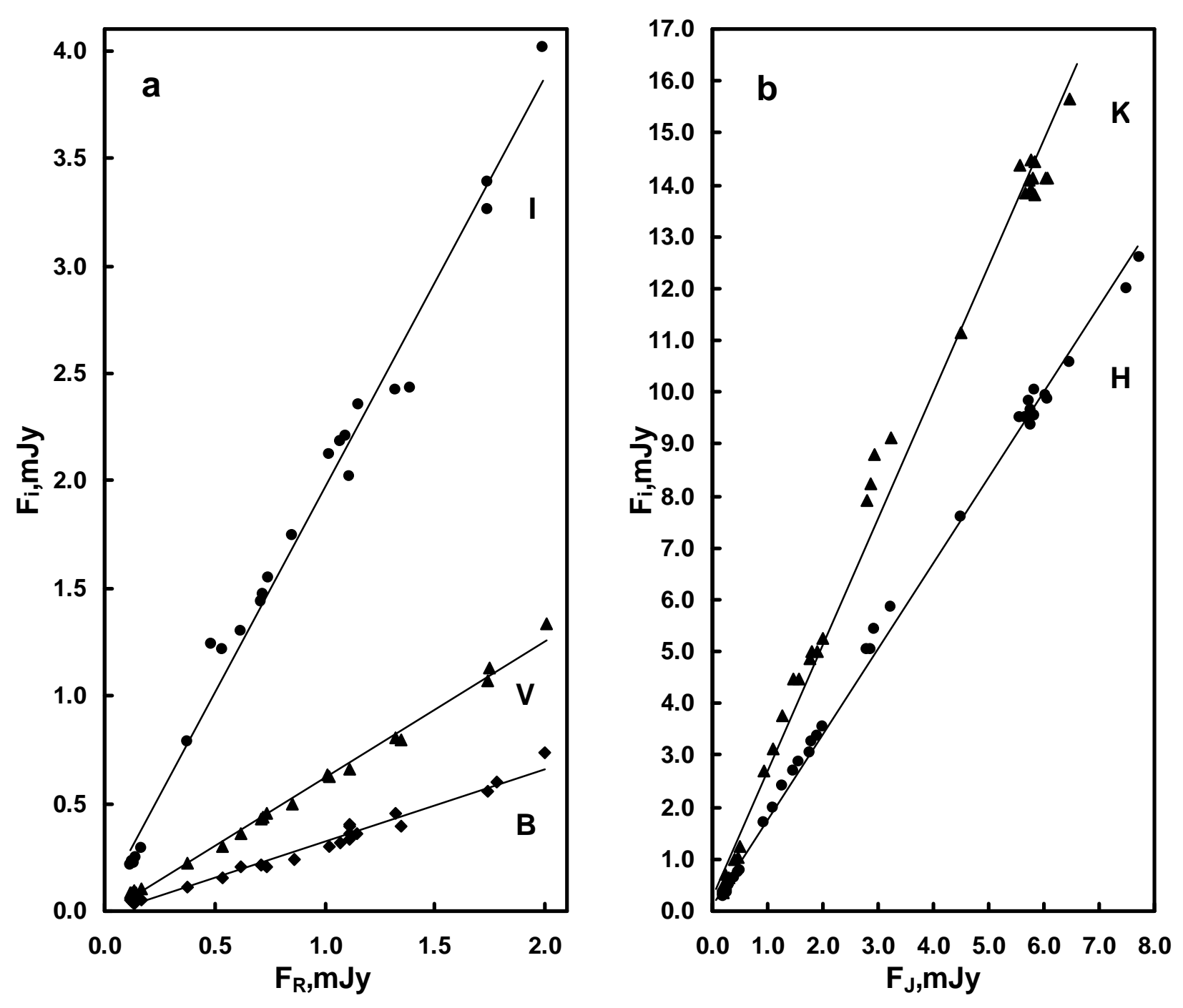

Fig. 6.- Flux-flux diagrams for optical (a) and (b) IR wavelengths. 


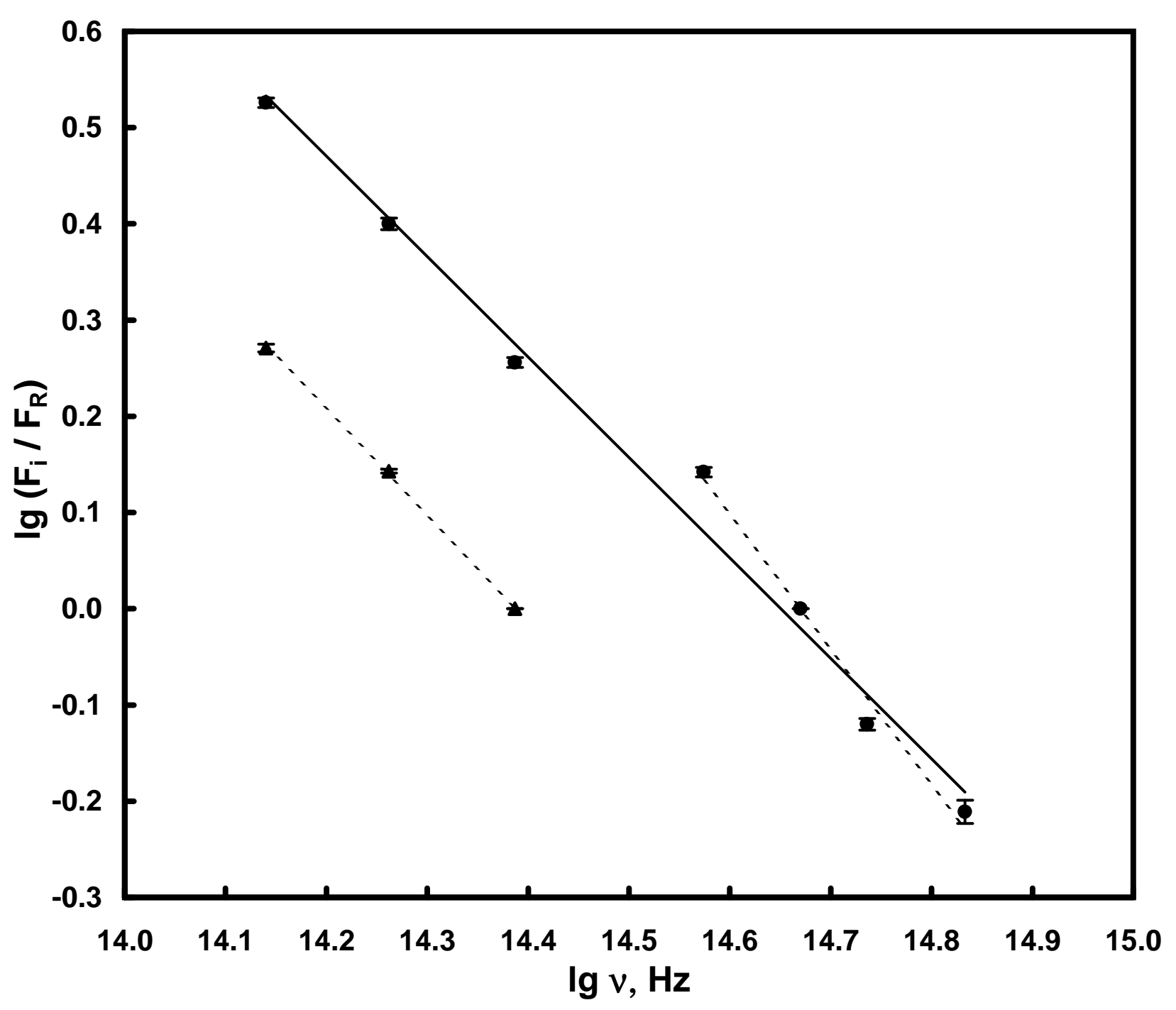

Fig. 7.- The relative SEDs at IR and optical wavelengths (dashed lines) and for the combined spectrum (solid line). The optical SED is relative to the $R$ band flux. The IR SED is relative to the $J$ band flux. The combined SED is constructed using a linear dependence between simultaneous observations in $J$ and $R$ bands. 


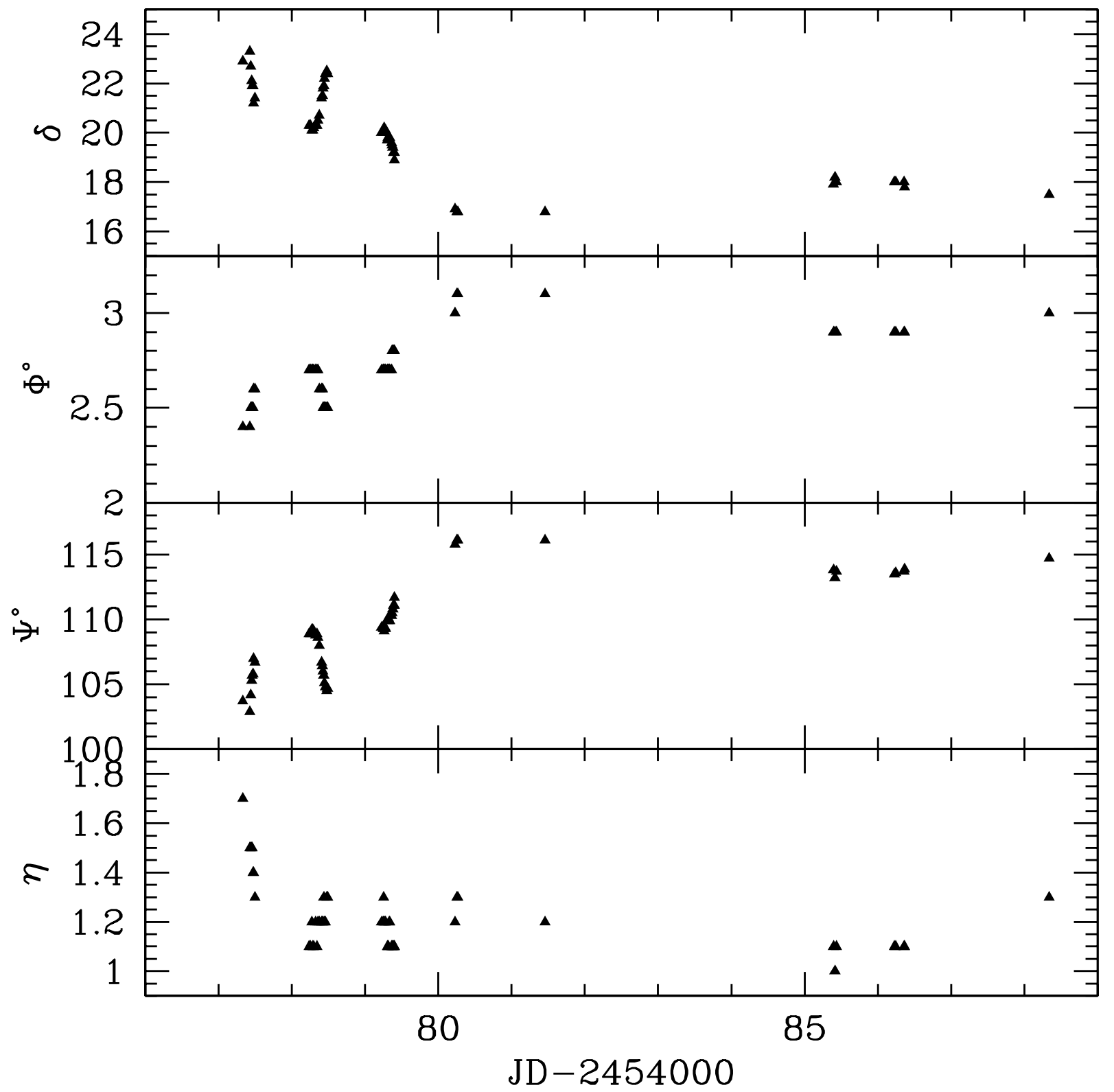

Fig. 8. - The derived values of Doppler factor, $\delta$, angle between the jet axis and line of sight, $\Phi$, viewing angle of the shock, $\Psi$, and compession factor of the shocked plasma, $\eta$. 